\title{
Mapeamento aero-geofísico de pegmatitos mineralizados na Província Pegmatítica de Alto Ligonha, no norte de Moçambique
}

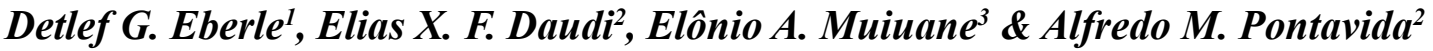

\begin{abstract}
Resumo No passado recente, uma extensa parte do território de Moçambique, foi coberta de levantamento aéreo de dados magnéticos e radiométricos de alta densidade. Desde assim, um valor, tem sido a preocupação da Direção Nacional de Geologia fazer a interpretação dos dados, acrescentando, assim,um valor adicional nos mapas aerogeofísicos para o uso das companhias mineiras. A Direção Nacional de Geologia (DNG) e a Universidade Eduardo Mondlane (UEM) num esforço conjunto com o Conselho de Geociência da Africa do Sul (CGS) fizeram o presente estudo dos campos pegmatíticos de Alto Ligonha, norte de Moçambique, dentro do Cinturão de Namama da era Meso-Proterozóico, para apoiar o sector de mineração da região. A integração de dados aerogeofisicos foi alcançada usando o alogaritmo média $\mathrm{k}$ aplicando a classificação não supervisionada dos dados obtida. A amostragem e avaliando por meio da análise discriminante para ganhar um critério de susceptibilidade da classificação obtida... A amostragem das concentrações de eTh e eK (em ppm) mais os dados da suscepetibilidade magnética aparente e da derivada vertical, resultaram num mapa de 850.000 vetores multivariados e um determinado número de classes. Este mapa ilustra claramente que há maior probabilidade dos pegmatitos mineralizados ocorrer nos afloramentos de gneisses anfibolíticos. A integração automatizada dos dados aero-geofísicos usando o alogaritmo média $\mathrm{k}$, provou ser um método rápido, direto e efetivo para compilação de mapa integrado com valor adicionado. A experiência adquirida com o estudo dos pegmatitos de Alto Ligonha encoraja a adoção desta nova metodologia para aplicar em outros locais do Cinturão de Moçambique. Assim, este método faria parte integrante do processo de mapeamento em Moçambique.
\end{abstract}

Palavras-chave: Aerogeofísica, integração de dados, classificação média k, análise discriminante, pegmatito, Cinturão de Moçambique.

\begin{abstract}
Airborne geophysical mapping of mineralized pegmatite in the Alto Ligonha pegmatite province, northern Mozambique. Major parts of Mozambique were recently flown to acquire high resolution airborne magnetic and radiometric data. Since then it has been the intent of the National Geology Directorate of Mozambique (DNG) to interpret these data generating value-added maps that are easier to use by the exploration and mining industries than mere airborne geophysical grid data and maps. DNG and Maputo-based EduardoMondlane University (UEM) entered a joint venture with the South African Council for Geoscience (CGS) to conduct a case study over the meso-Proterozoic Alto Ligonha pegmatite province in northern Mozambique to support the regional mining sector. Integration of the airborne geophysical data was achieved using the K-means algorithm for explorative unsupervised crisp clustering of the airborne geophysical data. Discriminant analysis was then used to obtain the degree of class membership of each sample previously classified by K-means. Prior to clustering, Th- and K-surface concentrations as well as apparent magnetic susceptibility and first vertical derivative data of the total magnetic intensity anomalies were determined to project a $100 \mathrm{~m}$-spaced grid comprising 850,000 multivariate sample vectors on the study area. Compilation of the classification results provided the value-added map illustrating that the probability to meet pegmatite bodies is highest where amphibole gneiss occurs. The automated integration of airborne geophysical data using the K-means algorithm is a fast, objective and efficient method to compile value-added maps. The experience made in the Alto Ligonha pegmatite province encourages the adoption of this new methodology over other parts of the Mozambique Fold Belt, becoming integral part of geological mapping ongoing in the country.
\end{abstract}

Keywords: Airborne geophysics, data integration, K-means clustering, discriminant analysis, pegmatite, Mozambique Fold Belt.

INTRODUÇÃO E MOTIVAÇÃo No âmbito do Projeto de Capacitação Institucional ao Sector de Recursos Minerais implementado entre 2001 e 2007, a Direção Nacional de Geologia (DNG) levou acabo o ma- peamento geológico do país que incluiu o levantamento aéreo de magnetometria e radiometria regional e de alta densidade. Atualmente o problema do setor geológicomineiro deriva se do fato de muitos recursos minerais

1 - Council for Geoscience, Pretoria, Gauteng, South Africa. E-mail: deberle@geoscience.org.za

2 - Direcção Nacional de Geologia, Maputo, Moçambique. E-mail: exfdaudi@gmail.com,pontamoz@gmail.com

3 - Universidade Eduardo Mondlane, Departamento de Geologia, Maputo, Moçambique. E-mail: muiuane@zebra.uem.mz 
serem descobertos esporadicamente pelos nativos e mineiros artenais sem nenhuma base geológica. O Governo de Moçambique está reunindo esforços para que o setor possa maximizar o valor da produção mineira.

O objetivo geral do projeto centra-se na demonstração como extrair e avaliar a informação dos dados aero-geofísicos de alta densidade para a exploração de recursos minerais tornando assim os mesmos mais atrativos aos investidores e contribuindo para a promoção da exploração e da indústria mineira em Moçambique.

Assim, a DNG em coordenação com o Departamento de Geologia da Universidade Eduardo-Mondlane (UEM) e o Conselho de Geociência (CGS) da Africa do Sul, elaboraram o projeto acima referenciado para extração de mais informações sobre os dados e acessar com mais detalhe a área em estudo. O projeto financiado pela agência sul-africana 'National Research Foundation' (NRF) decorreu num período de dois anos nos Distritos de Alto Molócuè e Gilé (Fig. 1).

Definiu-se como pre-requisito para atingir este objetivo, a capacitação de técnicos moçambicanos competentes para o uso das ferramentas e interpretação dos dados. Dai que o projeto previa a componente de formação de técnicos seniores e juniores.

A DNG como serviços geológicos do país faz a gestão não só de dados aéro-geofísicos, mas também de dados geoquímicos e de sensoriamento remoto. A integração desses dados deverá ser uma das componetes importantes. $\mathrm{O}$ estudo realizado no âmbito deste projeto, nos pegmatitos de Alto Ligonha, constitui um dos primeiros estudos conhecido de Africa meridional. Estudos bem semelhantes foram executados na década passada por vários autores brasileiros (Cainzos et al. 2002, Teixera et al. 2006, Silva 2009) utilizando a técnica de classificação não supervisionada conhecida com média k. Silva (2009) aplicou esta técnica para pesquisa de jazidas de tantalita e berilo na Província de Borborema, no Brasil. Por entanto, nós inserimos o conjunto de todos os dados aero-geofísicos ao processo da classificação numa só etapa obtendo como resultado um mapa pseudo-geológico que expõe a distribuição das várias classes na área de estudo. Além disso, executamos a análise discriminante 'aposterior' para verificação da probabilidade estatística

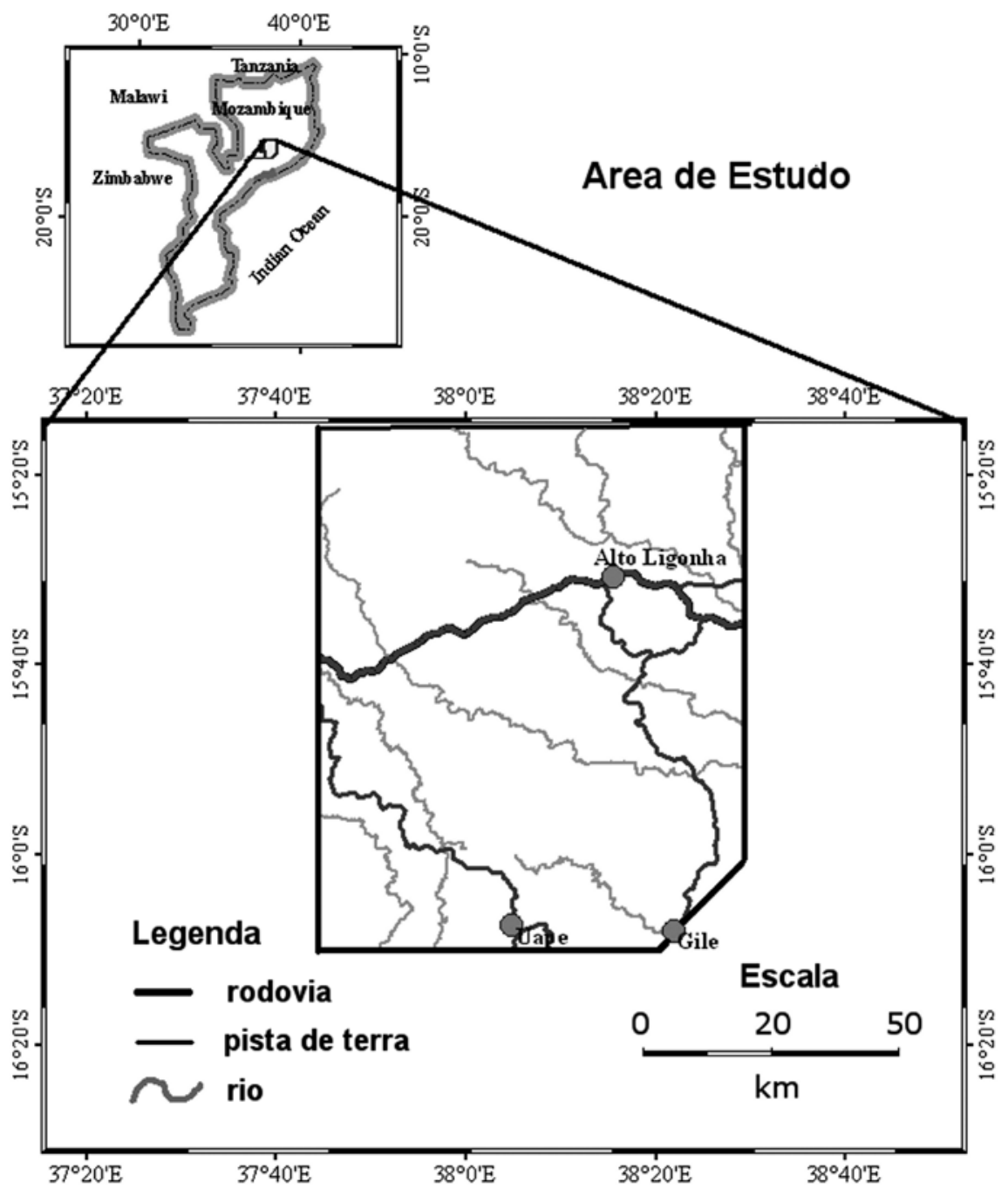

Figura 1 - Localização da área de estudo. 
do agrupamento de cada um vetor multivariado, pela qual a nossa metodologia destaca-se sobre os estudos acima mencionados.

Os objetivos preconizados neste estudo, foram alcançados apartir da análise e interpretação dos dados aeromagnéticos e radiométricos assim como os dados registrados durante o levantamento geofísico terrestre. A automatização total e integração dos dados (Eberle 1992, 1993; Stettler et al. 1998) baseada na técnica de classificação decidida ('crisp') e não-supervisionada (p.ex. Späth 1983, Deichsel \& Trampisch 1985) foi aplicada com sucesso na delimitação dos campos pegmatíticos. Realizou-se o levantamento de perfís perpendiculares aos corpos pegmatíticos e depósitos de tantalite e ouro conhecidos na região para verificação das anomalias interpretadas nos dados aéro-geofísicos e a correlação com as mineralizações.

GEOLOGIA E MINERALIZAÇ̃̃o As datações radiométricas evidênciam que a geologia da área foi afetada por dois cíclos metamórficos importantes, e que o último evento orogénico no embasamento de Moçambique a norte do Rio Zambeze é da idade próxima de $1 \mathrm{Ga}$ (Bingen et al. 2009), ou seja Kibariano (evento Luriano) segundo a terminologia adoptada pelo 'Bureau de Recherches Géologiques et Minières' (BRGM) da França (Jourde 1983).

Posteriormente, houve um 'evento térmico' (PanAfricano), ou seja um aquecimento disperso da crosta, o qual foi acompanhado pela instalação de granitos e pegmatitos metalíferos mas não por deformação importante.

A geologia da área de estudo é coberta pelo Cinturão de Moçambique, ocorrendo formações do Namala/ Cavarro, Murrupula Suite, Grupo de Molócuè e Morrua, Granitoides de Culicui e Gnaisses de Mocuba (Fig. 2).

Em termos litológicos, a área de estudo compreende uma sequência de rochas supracrustais ortognaíssicas, granodioríticas, tonalíica-trondjemíticas do Mesoproterózoico para além de ortognaisses graníticas, metavulcanos e granitos do Paleózoico Inferior. Os ortognaisses migmatíticos de Mocuba, poli-deformados, formam provavelmente a base supracrustal onde depositaram-se as rochas do Grupo de Molócuè. O Grupo de Molócuè representa uma sequência de camada intermédiaria de rochas metapelítica, calc-silicato e félsica, de gnaisses metavolcânicas máfica e ultrámafica supracrustais e localmente cortadas por Namama Thrust Belt. O Suite do Culicui consiste de granitos migmatíticos a não migmatíticos e de gnaisses e leuco-granitos augen (Cadoppi et al. 1987, Kröner et al. 1997, Direção Nacional de Geologia \& Council for Geoscience 2007).

A Formação de Morrua compreende a intercalação de magnetita com vulcanitos máficos a ultramáficos, quartzitos e quartzitos ferroginosos.

Os gnaisses Mesoproterózoicos foram mais tarde deformados durante a intrusão dos plutões Panafricanos e diques do Suite de Murrupula. Durante este período, duas gerações de pegmatitos intrudiram as rochas do Supergrupo de Nampula (Lächelt 2004). Verifica-se uma estreita relação lito-estratigráfica na distribuição dos pegmatitos do Grupo de Molócuè.

$\mathrm{Na}$ região em estudo ocorrem pegmatitos com o seu maior eixo em concordância estrutural com a xistosidade regional ou divergindo dela por pequeno ângulo (Maidge - direções médias $\mathrm{N}^{\circ} 5^{\circ} \mathrm{E}$ ) e pegmatitos acentuadamente discordantes ainda que, quase sempre, com direções do mesmo quadrante (Nahora - pegmatito com orientação $\mathrm{N}-\mathrm{S}$ e xistosidade $\mathrm{N} 55^{\circ} \mathrm{E}$ ).

Depósitos minerais Os pegmatitos de Alto Lingonha enquadram-se na área em estudo. Esta área é economicamente rica em pegmatitos que hospedam tantalite, terras raras com minerais como columbo-tantalite, berílio com minerais de lítio e gemas para além de ocorrências de ouro.

A presença de pegmatitos com colombo-tantalite é principalmente marcada por faixas brancas de quartzo leitoso esmagadas sobre a superfície da rocha metamorfizada. A espessa camada de alteração que cobre as rochas da superfície torna difícil efetivação de plenos estudos geológicos nas áreas pegmatíticas.

O principal campo pegmatítico com ocorrência de ouro ocorre em Morrua encaixado nas rochas da Formação de Morrua (Grupo de Molócuè) que é constituida por anfibolitos, gneisses, piroxenitos com granada e granulitos máficos (Lächelt 2004). O ouro aluvionar ocorre nos rios tributários de Namarroe e Molócuè. Ocorre também ouro primário em filões de quartzo associado ao ferro bandeado dentro dos anfibolitos de Morrua.

DADOS AERO-GEOFÍSICOS DE ALTA DENSI-

DADE Os dados aero-magnéticos e -radiométricos foram adquiridos em 2004, no norte de Moçambique, com espassamento entre linhas de vôo de $300 \mathrm{~m}$ e altitude de vôo de $100 \mathrm{~m}$. A direção dos perfís tinha uma orientação NW-SE cortando aproximadamente em perpendicular as unidades geológicas. A imagem ilustrando as anomalias do campo magnético total, (veja Fig. 3), espelha a orientação geral (SW-NE) dos corpos pegmatíticos, na zona central, uma estreita faixa com alta intensidade magnética.

A figura 4 mostra a composição falsa-cor no espaço RGB dos elementos radioativos de potássio ( $\% \mathrm{~K}$ 40), tório (ppm eTh) e urânio (ppm eU). Pode-se observar claramente a maior frequência de tório na zona norte oeste e a predominância de potássio no sul este. Através destes dados nota-se claramente a divisão do cinturão de cavalgamento do Namama (NTB). O Namama identifica-se pela cor escura que se encontra dentro da área predominada pelo potássio, no entanto, com o processo de metassomatismo houve perca de potássio, isto é substituição do potássio por outros elementos. É nesta faixa onde se encontra a maior parte das mineralizações de pegmatitos (Fig. 4).

INTEGRAÇÃO DE DADOS Foi aplicado um método rápido para integração objetiva de dados usando classificação não supervisada (Eberle et al. 2005, Eberle et al. 2009). Em termos tecnológicos, neste estudo, a integração automatizada objetiva usa a técnica média k para obtenção de grupos estatisticamente significantes apar- 


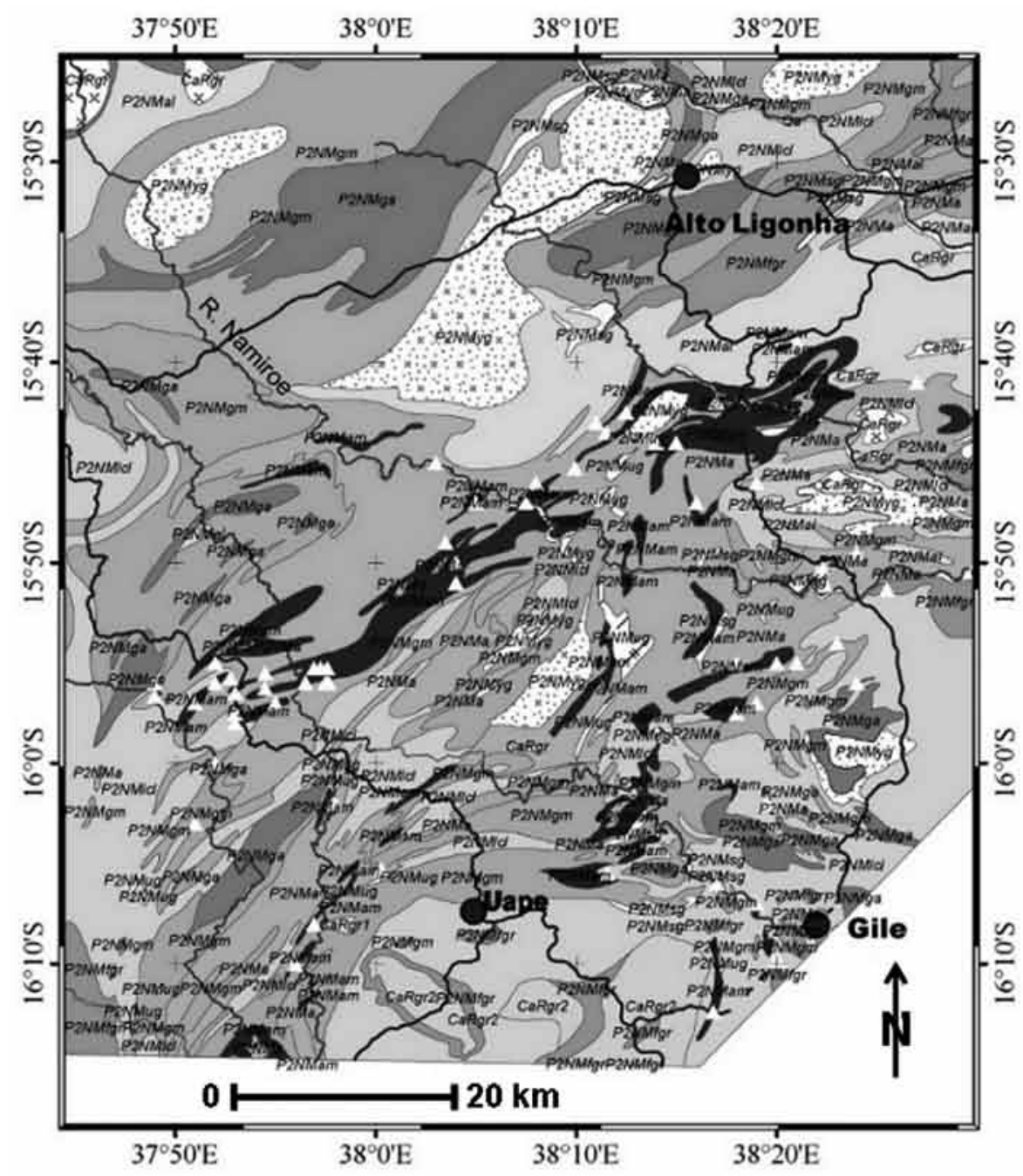

\section{LEGENDA}

P2NMam

Gnaisse anfibolítico, anfibolita granada (P2NMam)

Gnaisse augen com charnoquite (P2NMga)

Gnaisse biotítico bandeado e migmatite (P2NMgm)

Dolorite colunar (Jrdo)

Granito (CaRgr)

Gnaisse leococrático (P2NMIcl)

Gnaisse granítico com megacristais (P2NMyg)

Quartzito, arcose e quartzito ferroginoso (P2NMsg)

Gnaisse leocrático listrado (P2Nmal)

Gnaisse granítico (potássico) muito folheado (P2NMfgr)

Gnaisse ultramáfico (P2NMug)

Gnaisse indiferenciado (P2Nma)

Granito porfírico com pequenos fenocristais (CaRgr1)

Granito porfírico com grandes fenocristais (CaRgr2)

Figura 2 - Geologia do Cinturão Namama (Departamento Nacional de Geologia \& Council for Geoscience 2007). Local com triângulos brancos indicando mineralizações pegmatíticas. 

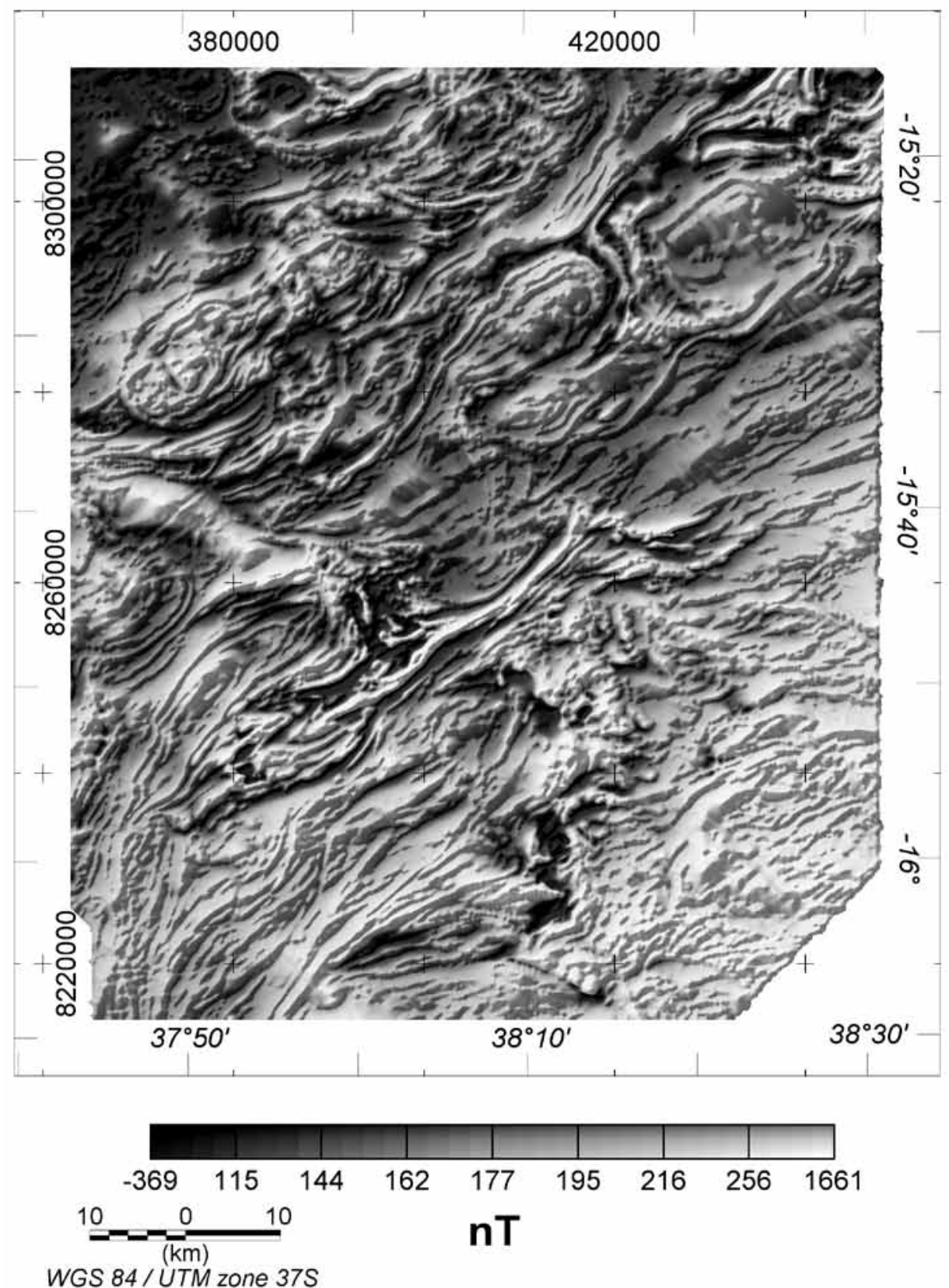

Figura 3 - Anomalias residuais $(\Delta T)$ do campo magnético na área de Alto Ligonha.

tir de dados aerogeofisicos com base no mapeamento geológico. Partindo da distância mínima (distância euclidiana) entre os vetores multivariados, o agrupamento calcula-se na base da média e do desvio-padrão dos mesmos. Logo que um vetor multivariado foi atribuido, permanece aí e não pode mudar de classe mais ('crisp clustering'). Uma medida para indicar a significância estatística ou qualidade da classificação de todos vetores multivariados (amostras) não é fornecida pelo método média k. Para compensar este defeito, aplicamos a ánalise discriminante linear após a classificação via média k. Baseada nesta classificação prévia, é a análise discriminante que fornece a medida de confidência para cada um vetor multivariodo através de uma classificação ‘a-posterior' pelo qual a nossa metodologia destaca- se. Assim, possibilita-se considerar a qualidade da classificação numa base quantitativa e, se for aconselhável, repetí-la com número de classes ou variáveis alterado. Além disso, pela análise discriminante, estabelece-se um conjunto de equações lineares que apresenta assinatura númerica padrão da área considerada.

Para obter o pseudo mapa geológico da área de Alto Ligonha, foram consideradas quatro variáveis. Nomeadamente foram usados os dados da susceptibilidade magnética e primeira derivada vertical dos dados magnéticos residuais $(\Delta \mathrm{T})$, e concentrações de potássio (\%) e tório (ppm). O intervalo amostrado foi de 100 metros resultando em 850.000 vetores multivariados.

O número adequado de grupos só pode ser determinado antes do processo de classificação pela estimativa 

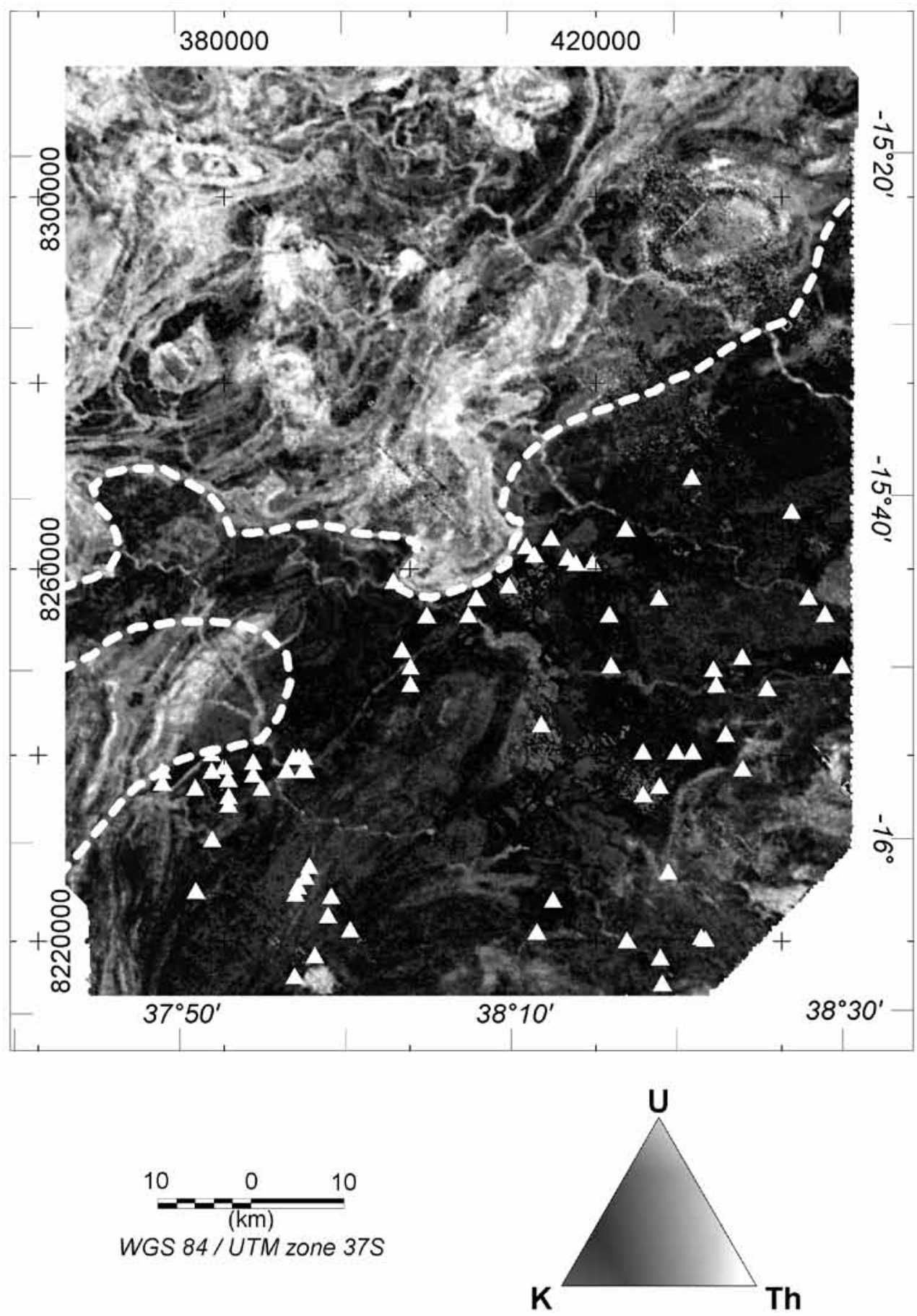

Figura 4 - Imagem ternária (composição falsa-cor) $R G B$ dos elementos radioativos, com a amplitude da contagem total $(n G y / h)$ superposta em tom cinza. Os triângulos em branco mostram as mineralizações dentro da faixa escura. As linhas tracejadas a branco delimitam os dois domínios radiométricos.

na base da geologia ou aplicando o método de indexação de Xie-Beni (Xie \& Beni 1991). O resultado da classificação está ilustrado na figura 5 , onde constam seis classes.

O desafio que se põe é identificar a associação de seis classes relacionadas com a geologia da área de estudo. Na figura 5 está refletida duma maneira holística a geologia/litologia da área de estudo, adicionada a informação geológica de profundidade pelos dados magnéticos. Pelo estudo integrativo da geologia mapeada (Fig. 2) e dos dados aero-géofísicos (Fig. 3 e 4) pode-se definir as seis classes em termos geológicogeofísicos (Tab. 1).

A tabela 1 reflete duma maneira geral a geologia da área de estudo. Na figura 5, a maioria das ocorrências minerais conhecidas (veja os triângulos a branco) é situada dentro das áreas atribuidas ás classes 1,2 e 6 . 

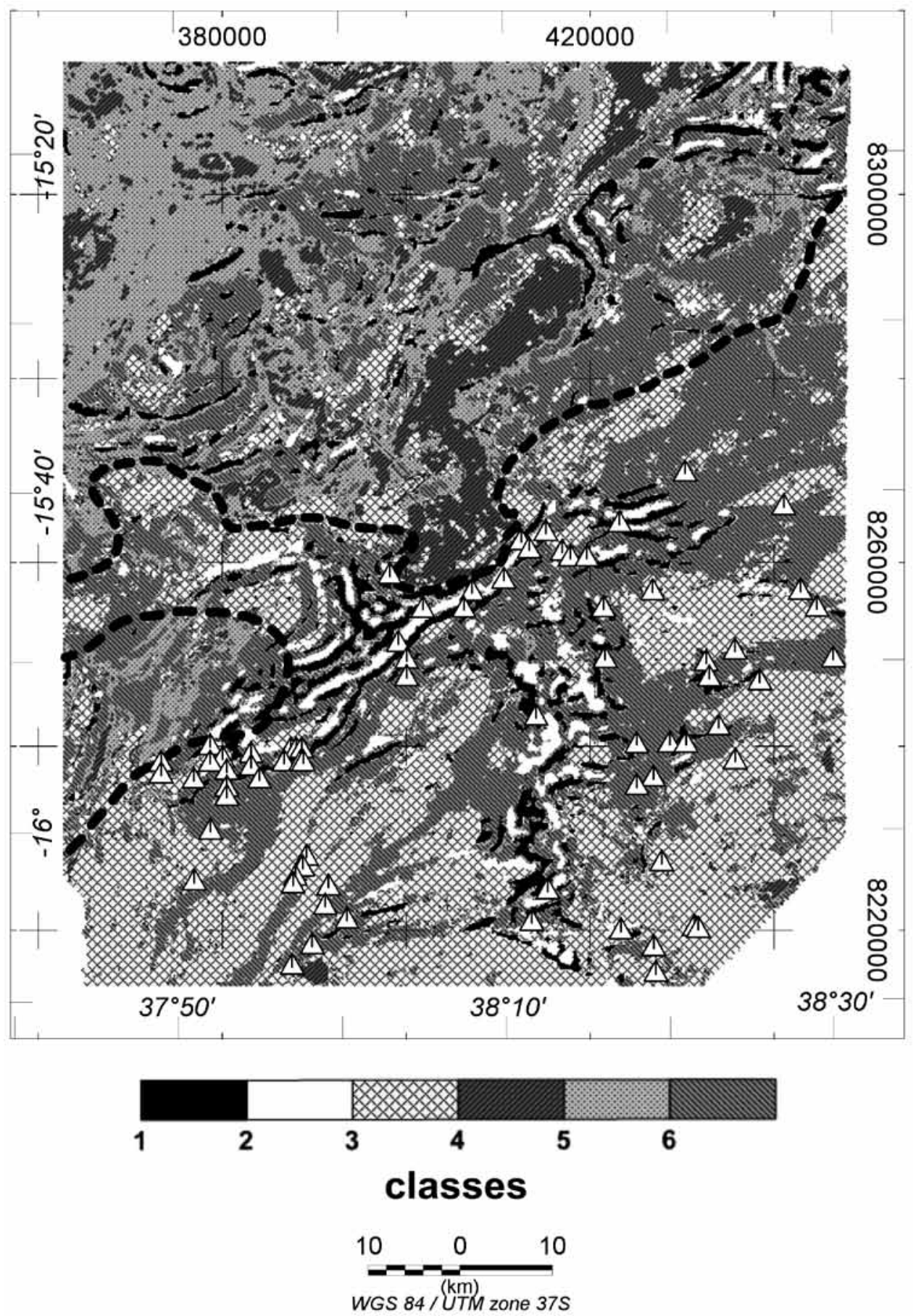

Figura 5 - Mapa de seis classes resultantes da classificação dos dados aerogéofísicos segundo a técnica de classificação não supervisionada média $k$ (K-means clustering). Os triângulos a branco mostram os corpos pegmatiticos mineralizados segundo o cadastro da DNG. As linhas tracejadas a preto delimitam os dois domínios radiométricos.

São estas classes que possuem potencial aumentado para exploração e mineração de novos corpos pegmatíticos.

Observando as figuras 4 e 5, é óbvio a extensão da classe 6 em ambos domínios radiométricos. Esta foi uma das razões que levou a experimentar o 'cluster analysis' para sete classes. Entretanto, o resultado não mostrou melhorias, sendo a nova classe 7 extensiva também para os dois domínios incluindo apenas relativamente poucas amostras, enquanto o número de amostras atribuidas a classe 6 mostrou-se pouco alterado e bastante estável também para o caso de sete classes.

Entretanto, o método de classificação automatizada revelou novas áreas promissoras para ocorrências de pegmatitos ainda não conhecidos naquela 
Tabela 1 - Atributos das litologias resultantes da integração dos dados aero-magnéticos e -radiométricos.

\begin{tabular}{l|l}
\hline Classe & Atributo de litologia/geologia \\
\hline 1,2 & $\begin{array}{l}\text { Gnaisses anfibolíticos, charnoquitos delimitando o } \\
\text { campo pegmatítico }\end{array}$ \\
\hline 3 & $\begin{array}{l}\text { Gnaisses leucocrastos em área de predominância de } \\
\text { potássio }\end{array}$ \\
\hline 4 & Gnaisses graníticos com megacristais \\
\hline 5 & $\begin{array}{l}\text { Gnaisses biotítico bandeados e migmatito em área } \\
\text { de predominância de tório }\end{array}$ \\
\hline 6 & Gnaisses leucocrasto Augen, charnoquitos \\
\hline
\end{tabular}

zona do cinturão de Moçambique apartir dos dados aero-geofísicos de alta densidade.

Para estabelecer um parâmetro de confidência no agrupamento (ou qualidade de sócio) individual de todos os vetores multivariados, foi feita uma classificação "a-posterior" através da análise discriminante linear, cujo resultado apresenta a entidade dos valores confidenciais de cada um dos vetores multivariados. Nas áreas coloridas a cinza branco da figura 6 , os vetores multivariados agruparam-se com uma significância (ou qualidade de sócio) de mais de $75 \%$ exprimindo agrupamento bem estável. O conjunto de equações lineares visando descobrir os pegmatitos ainda subjacentes

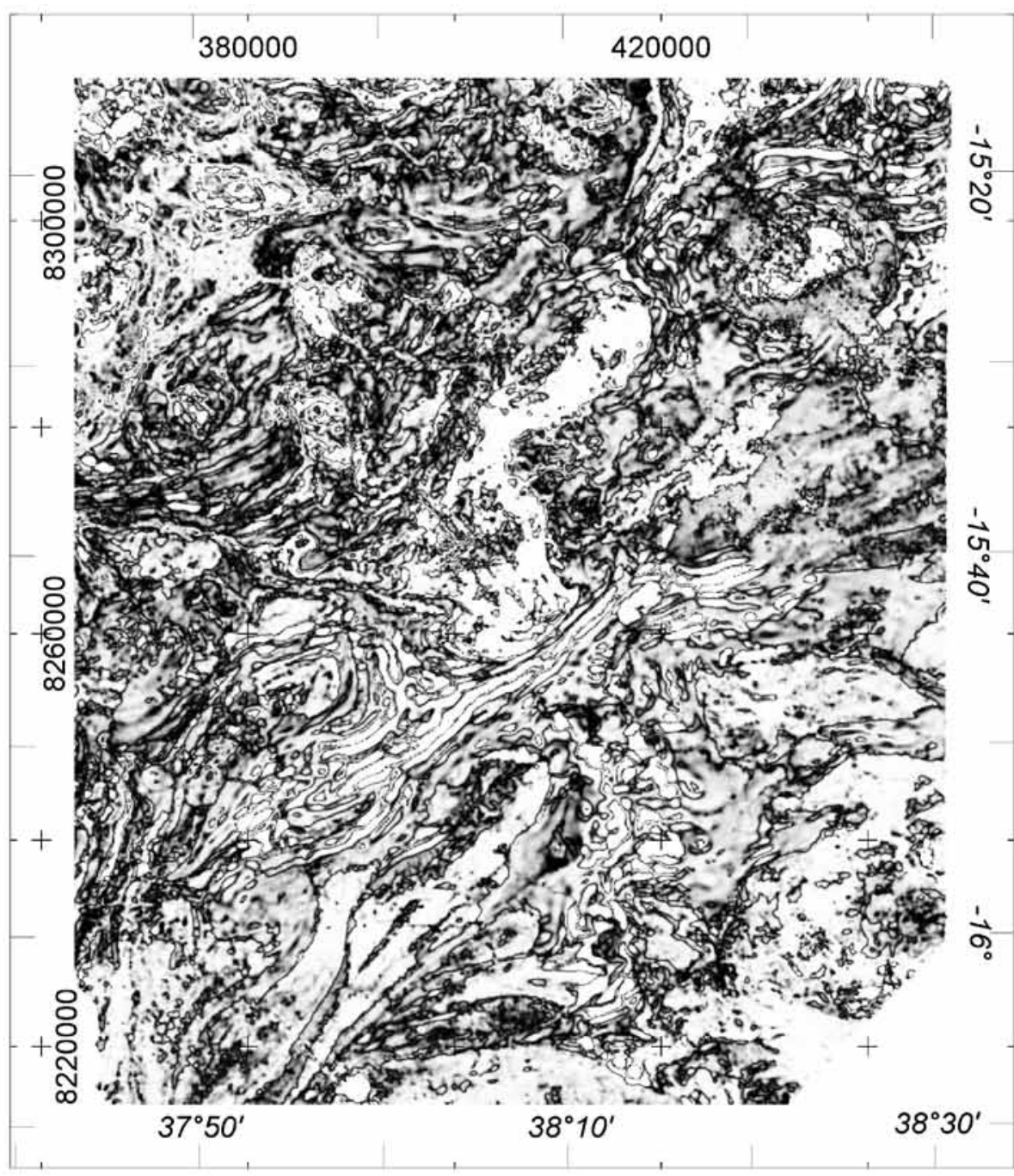

\section{Qualidade de Sócio \\ (Degree of Class Membership)}

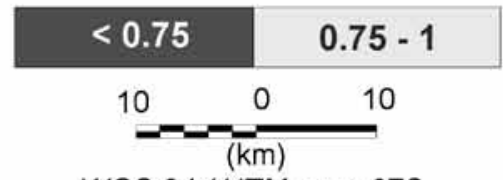

WGS 84 / UTM zone $37 S$

Figura 6 - Mapa mostrando a confidência do agrupamento dos vetores multivariados. 
nas condições geológicas do cinturão de Moçambique, constitue mais um resultado da análise discriminante.

LEVANTAMENTO GEOFÍSICO TERRESTRE Durante o trabalho de gabinete, foram identificadas anomalias quer aero-magnéticas quer aero-radiométricas que conduziram na selecção das áreas potenciais alvos para a pesquisa terreste mais detalhada.

A verificação e apuramento em detalhe das anomalias aero-magnéticas e -radiométricas foi realizado no terreno com abertura de perfis seguindo estradas terciárias associando com a litologia adjacente a estrada. Outros perfis foram levantados em depósitos de tantalite, ouro e gemas já conhecidos na área para estudar a relação entre as observações geofísicas e as mineralizações.

Foram registradas leituras da intensidade magnética total, da conductividade eléctrica aparente (pelo equipamento EM-34) e da radiação dos rádio-elementos naturais $40 \mathrm{~K}$, Th e U. O levantamento magnético sempre se realizou após o estabelecimento da estação de base para a correção diurna dos dados magnéticos. As estações efetuadas nos perfís registrados ao longo das picadas tinham intervalo de 15 metros, enquanto que nos depósitos o espaçamento era de cinco 5 metros. Enquanto, as leituras da radiação gama foram efetuadas numa distância de 10 metros entre si. O levantamento da condutividade aparente foi feito com uma distância de separação de 20 metros entre as bobinas transmissora e receptora na posição vertical-coplanar. O intervalo das leituras era também de $15 \mathrm{~m}$ ao longo das picadas e de $5 \mathrm{~m}$ nos depósitos e na vizinhança imediata deles.

Depois da avaliação de todos os perfis, chegouse a conclusão que a magnetometria forneceu os resultados mais favoráveis a respeito da localização de corpos pegmatíticos, na área de estudo, adjacente a rocha encaixante bastante magnética do típo anfibolítico ou charnoquito. Além disso, foram observadas algumas anomalias magnéticas de magnetização inversa (Fig. 7) na vizinhança imediata dos corpos pegmatíticos.

Da ánalise dos registos electromagnéticos pode-se também concluir que a condutividade aparente aumenta sobre os corpos pegmatíticos por um factor de 4 a 8 (Fig. 7). Os resultados menos indicativos resultam das medições da radiação gama, provavelmente por causa da radiação ambiental muito baixa bem como do intervalo relativamente curto de apenas 30 secundos em cada estação medida.

CONCLUSÕES As altas anomalias magnéticas e baixos conteudos radiométricos coincidem com rochas mineralizadas já conhecidas no Cinturão de Namama e Lúrio sugerindo que os pegmatitos de gemas estão espacialmente associados a rochas máfica-ultramáficas e anfibolitos como pode-se observar no Gilé e Morrua. Em contraste, os pegmatitos com mineralizações coincidindo com valores altos de potássio e baixa intensidade magnética, caracterizam rochas graníticas ou gnaisse granítico localizados mais a sul da área de estudo onde ocorrem os pegmatitos de Uape e Marropino.

A distribuição dos pegmatitos observados da integração dos dados aero-geofisicos revelam um controle estrutural pelos dois maiores cinturões e confinados a gneisses anfibolíticos, mas ocasionalmente ocorrem em gneisses graníticos.

A integração automatizada em combinação com observações de campo incluindo a disponibilidade de vários dados usados neste estudo estabeleceu com sucesso uma metodologia para identificar campos pegmatíticos na região de Alto Ligonha, norte de Moçambique. Pode-se confirmar que a metodologia da classificação decidida e não-supervisionada via média $\mathrm{k}$ combinada com a análise discriminante, aplica-se com sucesso para delimitar estruturas e unidades geológicas que controlam a distribuição espacial de campos

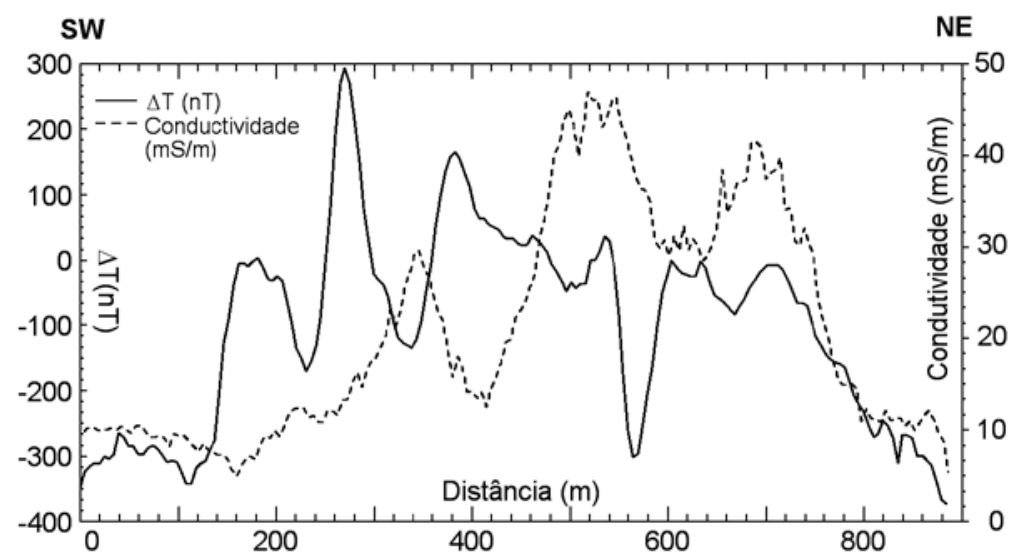

Figura 7 - Perfis terrestre de magnetometria e electromagnometria atravessando o corpo pegmatítico. Os xistos anfibólicos estão associados com anomalias magnéticas no intervalo entre 500 a $600 \mathrm{~m}$. Flanqueam imediatamente os corpos pegmatíticos mineralizados a $S W$ da anomalia magnética. 
pegmatíticos no cinturão de Moçambique.

Os resultados alcançados encorajam para extendermos este princípio da integração automatizada de dados não se baseando apenas nos dados aero-geofísicos como variáveis, mas também fazendo o uso dos dados geo-químicos e das imagens de satélites.

Agradecimentos Os autores deste trabalho expressam seus agradecimentos ao 'National Research Foundation of South Africa (NRF)' que viabilizou recursos para execução da investigação, dentro do programa do 'South African Regional Cooperation Fund for Scientific Research and Technological Development' bem como ao Conselho de Geociência da Africa do Sul (CGS), a Direcão Nacional de Geologia de Moçambique (DNG), a Universidade Eduardo Mondlane (UEM) de Maputo e a Direção Provincial de Recursos Minerais da Zambézia por terem aceitado este desafio. Também agradecemos ao árbitro para várias sugestões como melhorar o manuscrito.

\section{Referências}

Bingen B., Jacobs J., Viola G., Henderson I.H.C., Skår Ø., Boyd R., Thomas R.J., Solli A., Key R.M., Daudi E.X.F. 2009. Geochronology of the Precambrian crust in the Mozambique belt in NE Mozambique, and implications for Gondwana assembly. Precambrian Research, 170:231-255.

Cadoppi P., Costa M., Sacchi R. 1987. A cross-section of the Namama Thrust Belt (Mozambique). Journal of African Earth Sciences, 6(4):493-504.

Cainzos R.L.P., Pascholati E.M., Souza Filho, C.R. 2002. Utilização da classificação não-supervisionada na integração e interpretação de dados geofísicos em Cuba centro-oriental. Revista Brasileira de Geofisica 20(3):151-161.

Deichsel G. \& Trampisch H.J. 1985. Clusteranalyse und Diskriminanzanalyse. Stuttgart, Gustav Fischer, 135 p.

DIRECÇÃO NACIONAL DE GEOLOGIA \& COUNCIL FOR GEOSCIENCE 2007. Noticia explicativa das folhas 1537, 1538, 1539, 1540, 1637, 1638 e 1639-40, escala 1:250,000; Pretoria.

Eberle D. 1992. Interpretation of multi-method geophysical borehole logging data from KTB Oberpfalz VB using multivariate statistical analyses. Scientific Drilling, 3:16-26.

Eberle D. 1993. Geologic mapping based upon multivariate statistical analysis of airborne geophysical data. International Institute for Aerospace Survey and Earth Sciences (ITC) Journal, 1993-2:173-178.

Eberle D., Cole J., Häuserer M., Stettler E. 2005. Combined stochastic and deterministic modeling as an innovative approach to jointly interpret multi-method airborne geophysical data sets. In: South African Geophysical Association, $9^{\text {th, }}$ Biennial Conference, Cape Town, Extended Abstract Volume, 4 p.

Eberle D., Cole P., Nyabeze P. 2009. Acquiring high resolution airborne geophysical data and recognition of new mineral exploration potential as part of a development program launched by the South African government. In: International Geophysical Conference, $20^{\text {th }}$ and Exhibition ASEG 09, Adelaide-SA, Conference Handbook, Preview, 138:71.
Jourde G. 1983. La chaîne du Lurio (Nord Mozambique): Un témoin de l'existence de chaînes kibariennes en Afrique Orientale. In: Colóquio sobre Geologia de Africa, 12, Tervuren, Bélgica, Abstrato, no. 50.

Kröner A., Sacchi R., Jaeckel P., Costa M. 1997. Kibaran magmatism and Pan-African granulite metamorphism in northern Mozambique: Single zircon ages and regional implications. Journal of African Earth Sciences, 25(3):467-484.

Lächelt S. 2004. The Geology and Mineral Resources of Moçambique. Maputo, Direcção Nacional de Geologia, $515 \mathrm{p}$.

Silva S.M.P. 2009. Espectroscopia de imageamento e gamaespectrometria aérea e terrestre de pegmatitos e granitos da porção sul da Província Pegmatítica da Borborema (PPB), nordeste do Brasil. Tese de Doutoramento, Instituto de Geociências, Universidade Estadual de Campinas (SP).

Späth H. 1983. Cluster-Formation und Analyse. Oldenbourg, München-Wien, 236 p.

Stettler E.H., Eberle D., Cole P. 1998. Multivariate statistical analysis and compact volume inversion in the semiautomatic construction of three-dimensional geological models from multi-disciplinary geophysical data sets. In: Geocongress '98, Pretoria, Expanded Abstract Volume, p. 140-143.

Teixeira A., Silva A.M., Pires A.C.B., Moraes R.A.V., Souza Filho C.R. 2006. Integração e análise de dados aerogeofísicos por meio da aplicação de técnicas de processamento digital de imagens e classificação não supervisionada: O exemplo do Greenstone Belt Rio das Velhas, Quadrilátero ferrífero, MG. Revista Brasileira de Geofisica, 24(4):559-572.

Xie X.L. \& Beni G. 1991. A validity measure for fuzzy clustering. IEEE Transactions on Pattern Analysis and Machine Intelligence, 13:841-847.

Manuscrito ID 16893

Submetido em 23 de fevereiro de 2010 Aceito em 01 de dezembro de 2010 